\title{
A Chiral fNIRS Spotlight on Cerebellar Activation in a Finger Tapping Task
}

\author{
G. Rocco*, J. Lebrun, O. Meste, and M.-N. Magnié-Mauro
}

\begin{abstract}
Functional Magnetic Resonance Imaging (fMRI) has been so far the golden standard to study the functional aspects of the cerebellum. In this paper, a low-cost alternative imaging, i.e. functional Near-Infrared Spectroscopy (fNIRS) is demonstrated to achieve successful measurements of the cerebellar hemodynamics towards the challenging observation of motor and cognitive processes at the cerebellar level. The excitation and reception optodes need to be properly placed to circumvent a major hindering from the shielding by the neck muscles. A simple experimental protocol, i.e. finger tapping task, was implemented to observe the subject's engagement and the presence of functional asymmetries. Marked differences among subjects with different levels of lateralization were clearly noticed in terms of activation and latencies, together with peaks in the hemodynamic response following neural activation. These preliminary results suggest also differences in the hemodynamic behavior between the brain and the cerebellum and encourage future and extended analysis in this direction.
\end{abstract}

Clinical Relevance-This establishes the possibility to use a novel technique (fNIRS) to study cerebellar hemodynamics instead of fMRI.

\section{INTRODUCTION}

Historically, the functional aspects of cerebellum have always been poorly characterized. Due to its remote location, and shielded by the neck muscles, it did not appear particularly suitable for clean and genuine signal recordings. However, the "little brain" contains more than a half of the total number of neurons in the brain, despite being only $10 \%$ of the total brain volume [1]. Thus, such a dense structure suggests a high level of complexity, which still needs to be further explored.

In fact, over the recent decades relevant advancements have been made to better understand the cerebellar functions. The cerebellum was only believed to play a pivotal role in motion control and smoothness, but recently it has been proved to be involved also in cognition and emotion. At the clinical level, this has been epitomized by the Cerebellar Cognitive and Affective Syndrome [2], i.e. a series of cerebellar-induced chronic pathological conditions, which arise with deficits in executive function, visuospatial cognition, spatial disintegration, language and speech, and emotion. With the non-motor role of the cerebellum being acknowledged, the objective is now to unravel the underlying mechanisms and its functional organization.

Up to now, mostly neuroimaging techniques, such as fMRI, were used to elucidate the functional role of the cerebellum in multiple tasks, which led to the development of

\footnotetext{
* Research supported by the European Union H2020 MSCA COFUND programme "BoostUrCareer" under grant agreement 847581.

* Corresponding author is G. Rocco - Email: grocco@i3s.unice.fr.
}

modern cerebellar systemic neuroscience [3]. In clinical practice, most of the cerebellar investigations focus on metabolic or hemodynamic processes which concern essentially pathological subjects with cerebellar impairments. However, as a finer temporal analysis of the cerebellar activity is desirable, the intrinsic nature of the hemodynamic and metabolic responses given by fMRI or PET may constitute a strong limitation. Especially, in a research experimental context, a finer temporal resolution become essential to correlate any measured cerebellar signal with behavioral parameters, e.g. reaction times, or to compare activation latencies between cerebellum and other brain structures and finally to assess putative fine-grained synchronization properties between nodes of the involved cerebral network and the cerebellum, whose activation is ten times faster [4]. Besides the issue of temporal resolution, the physical constraints of the acquisition device must be considered. For example, a fMRI or PET study requires the subject to lay in a narrow scanner and to perform tasks in a very different condition from the normal life, by posing limitations on movement, hearing, and the variety of tasks that can be demanded. Also, multimodal approaches like with electroencephalography (EEG) or ultrasonic imaging (USI) get quite tedious to implement.

In this perspective, the last decades have seen the advent of functional near-infrared spectroscopy (fNIRS) as an emerging technology in cognitive neuroscience [5]. fNIRS is an optical technique that allows to measure the changes in concentration of oxygenated $\left(\mathrm{HbO}_{2}\right)$ and deoxygenated $(\mathrm{HbR})$ hemoglobin caused by neuronal activation in the brain tissue, by exploiting the transparency of the biological tissue within the near-infrared range. fNIRS appears particularly advantageous because it is non-invasive, cost-effective, silent, and relatively comfortable; it also exhibits finer temporal resolution than $\mathrm{AMRI}$ and potentially higher spatial resolution than EEG; it can be portable, thus suitable for different kinds of experimental protocols.

To the best of our knowledge, no investigation of the cerebellar functional aspects with fNIRS has been achieved yet. Hence, considering the outstanding advantages of this technology and the promising results of our recent feasibility study [6], the aim of the present work is to assess the capability of fNIRS to describe the cerebellum response of subjects with different levels of lateralization during a finger tapping task.
All authors are with Université Côte d'Azur, Nice, France. G.R, J.L, O.M are with lab. I3S (CNRS/UCA); M.N.M.M. is with lab. BCL (CNRS/UCA) and $\mathrm{CHU}$ Nice. 


\section{MATERIAL AND METHODS}

The goal of our experimental recordings is to obtain a fNIRS measurement of the so-called blood oxygenation level dependent (BOLD) effect, upon which fMRI studies also rely in clinical practice. Specifically, at the brain level, neural activation is associated with an increase in local arterial vasodilation, which leads in turn to a regional increase in cerebral blood flow and volume and thus oxygenation.

\section{A. Experimental design}

For this study three subjects (mean age $=31$ ) were recruited. Each person was asked to sit on a chair in a dark room, to limit interferences with measurements in the ambient light and to reduce the activity of the visual cortex, which is closely located to the cerebellum. First, a 2-minutes baseline recording was done. Then, a 4-minutes experimental session followed: six consecutive 40 -seconds trials, i.e. 10 seconds of activity (finger tapping) followed by 30 seconds of rest. The whole experiment was repeated twice, respectively for the left and the right hand. The subjects were asked vocally by a speaker to tap the finger at the desired moment. A delay of 1 or 2 seconds can be considered negligible for the sake of the experiment and for the physiological hemoglobin recovery time [7]. In particular, the experimental protocol is defined in such a way to observe eventual functional asymmetries between the movement of one finger and the controlling cerebellar hemisphere. Each subject filled in a handedness questionnaire whose score spans from -100 (fully left-handed) to 100 (fully right-handed) [8]. The data acquisition was performed in compliance with laws and institutional guidelines and was approved by the UCA's CERNI (Comité d'Ethique pour les Recherches Non Interventionnelles).

\section{B. Data acquisition}

The Artinis Oxymon Mk III system was used for data collection. The employed wavelengths were 763 and $858 \mathrm{~nm}$, and the sampling frequency $50 \mathrm{~Hz}$. Two channels (source and detector) were acquired, respectively on the left and right cerebellar hemisphere. The source-detector distance was set to $35 \mathrm{~mm}$, in order to ensure an adequate depth to access the real cerebellar signal and to elude the splenius muscle and extracerebral activity above a bony triangular part. The optodes were placed in two reference positions, namely CB1 and CB2, that were added to the conventional EEG 10/20 system [9], as shown in Figure 1.

\section{Data analysis}

All the analyses were performed using Matlab and the Fieldtrip toolbox [10]. First, an assessment of the signal quality with a frequency domain analysis was carried out, to verify the appropriate neurovascular coupling. The Power Spectral Density (PSD) of the signal was computed using the Welch periodogram with a window length of 120 seconds and an overlap of 50\%. After, the measured light intensities were used to assess the local oxygenation concentration through the modified Lambert-Beer law [11]: in order to remove the effect of scattering, water and other non-related components, the first attenuation measurement is subtracted to the subsequent ones with the result of a change in attenuation which is directly dependent on the change of concentration of the chromophores $\left(\Delta \mathrm{HbO}_{2}\right.$ and $\left.\Delta \mathrm{HbR}\right)$. Then, the signals were visually inspected to detect motion artifacts. The initial baseline was subtracted

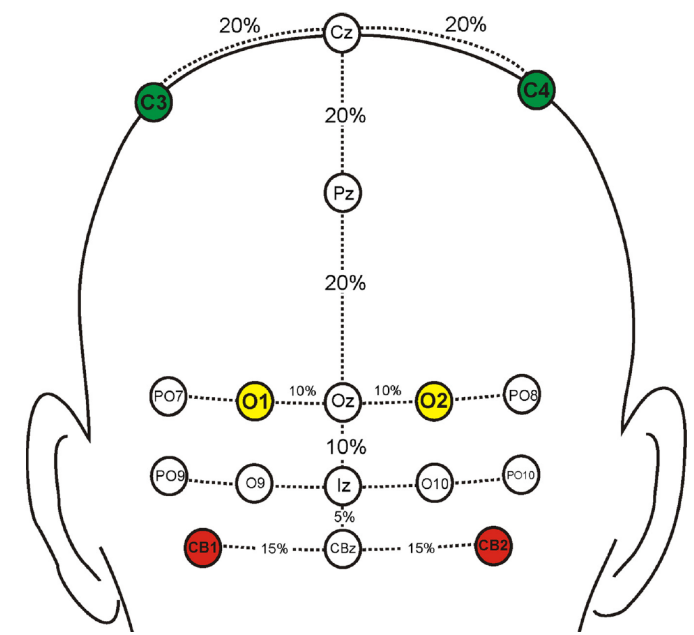

Figure 1. $\mathrm{CB} 1$ and $\mathrm{CB} 2$ optodes placement (in red) with respect to the $10 / 20$ system [9]

to each signal. A band-pass FIR filter with order 1000 and cutoff frequencies $0.01-0.09 \mathrm{~Hz}$ was applied [11]. Then, the time-locked average of the trials was calculated for each subject and each experimental session.

\section{RESULTS}

Fig. 2 shows a short segment of the raw fNIRS signals acquired from both channels and displayed with the Fieldtrip toolbox. The heart rate signal can be clearly observed, thus proving adequate optodes coupling with the skull. This is confirmed also by the PSD of the fNIRS signals (an example in Fig. 3), where it is possible to identify the heart rate component, the respiration component, and the Mayer wave component, which are then subsequently removed by means of the band-pass filtering. An example of filtered signal is illustrated in Fig. 4 (S2, left finger tapping): a marked difference can be noticed between the baseline period and the experimental session, (signaled by the vertical black dashed line), where the responses are higher in amplitude and appreciably synchronized. The results of the time-locked average of the trials for all the subjects and the sessions are reported in Fig. 5. The first column shows the averaged responses of CB1 (left hemisphere), while the second column

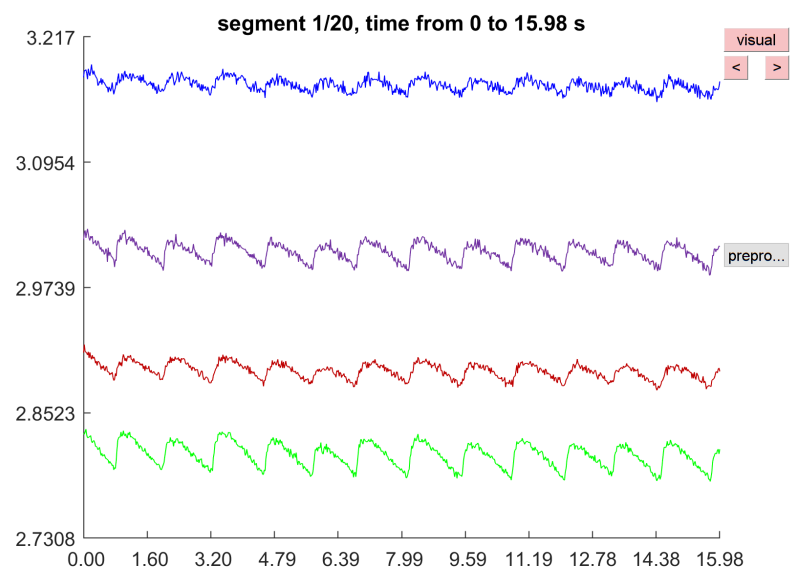

Figure 2. Segments of raw fNIRS signal $\left(\Delta \mathrm{HbO}_{2}\right.$ and $\left.\Delta \mathrm{HbR}\right)$ for both hemispheres 


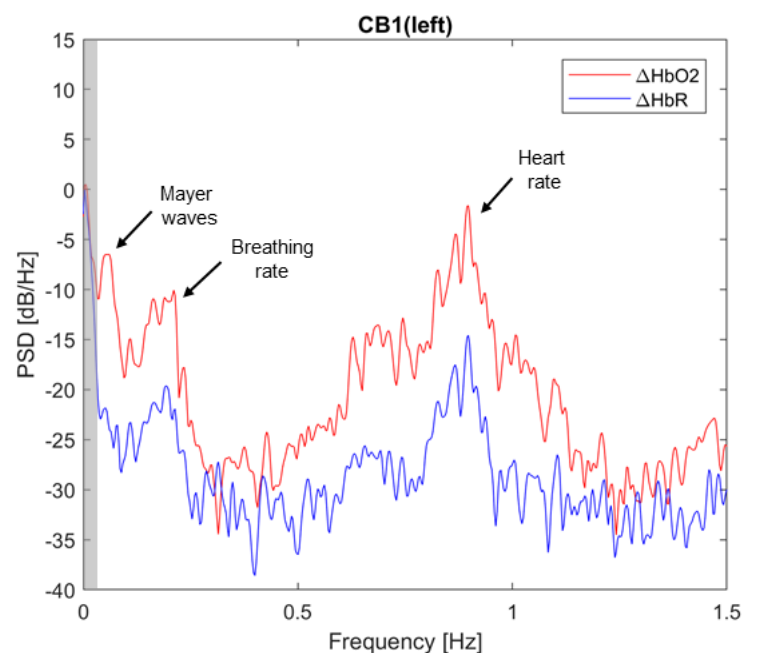

Figure 3. An example of PSD of fNIRS signal from CB2 position. The grey shaded area is the NIRS signal of interest.

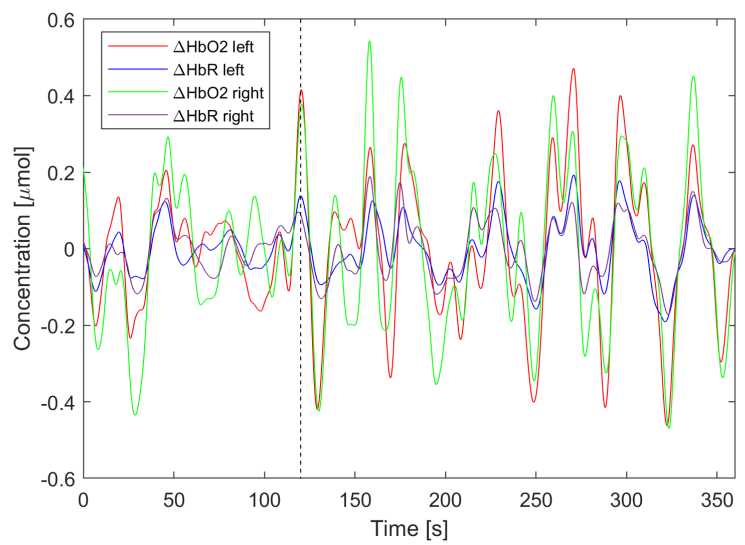

Figure 4. An example of filtered fNIRS signal. The vertical dashed black line marks the end of the baseline and the start of the session

CB2 (right hemisphere). Each 4-figures subplot is referred to one subject and represents in the first row the results of left finger tapping and in the second row the right finger tapping. To correctly interpret the results, it is necessary to consider the scores of the lateralization questionnaire, respectively 70 for S1 (right-handed with unusual tendencies towards left), 100 for S2 (full right-handed), and -90 for S3 (full left-handed). For this reason, the high variability of the responses betweensubjects was expected in terms of shape, latency, and amplitude. In particular, looking at the left finger tapping, S1 and $\mathrm{S} 3$ showed slower ipsilateral activation after the end of the motor task (marked by the vertical dashed black line), while S2 showed a fast and sharp increase of $\Delta \mathrm{HbO}_{2}$ in both hemispheres. This behavior is confirmed also by the PSD of the filtered signal: a peak occurs in correspondence of the stimulation frequency $\sim 0.025 \mathrm{~Hz}(1 /(10 \mathrm{~s}$ task $+30 \mathrm{~s}$ rest $) \mathrm{Hz})$ in CB2 for S2 (Fig. 6) and CB1 for S3 (Fig. 7). In fact, the NIRS signal is rather uncorrelated to the pace of the activity, and strictly dependent on the experimental block duration. Concerning the right finger tapping, a classical bell-shaped response can be noticed ipsilaterally in S2, while a very low intensity one in S3. A peculiar dynamic is observed in S1, who showed an anticipated activation before the task that could be attributed to a learning effect or a sort of automatic behavior. In general, S1 exhibited a task-dependent behavior unlike the
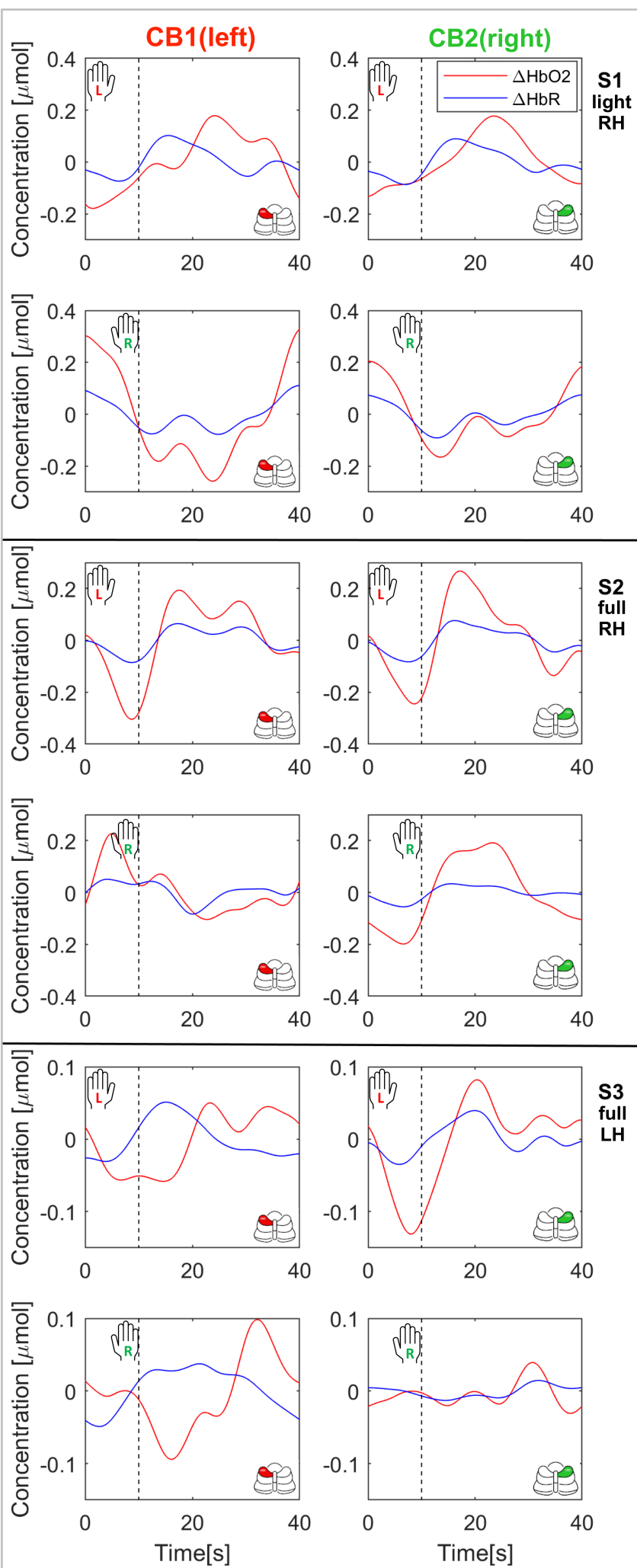

Figure 5.The time-locked average response for all the subjects and sessions. The vertical dashed black line marks the end of the tapping. 4 figures per subjects with the first row showing the results of left finger tapping and the second row the right finger tapping.

other two subjects: $\mathrm{CB} 1$ and $\mathrm{CB} 2$ responses are very similar within the same task and tend to adapt similarly to a change of motor activity. 

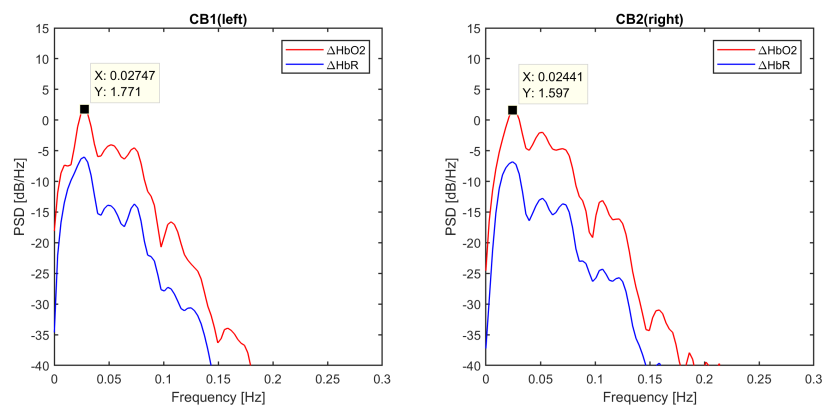

Figure 6. PSD of the left finger tapping signal from S2
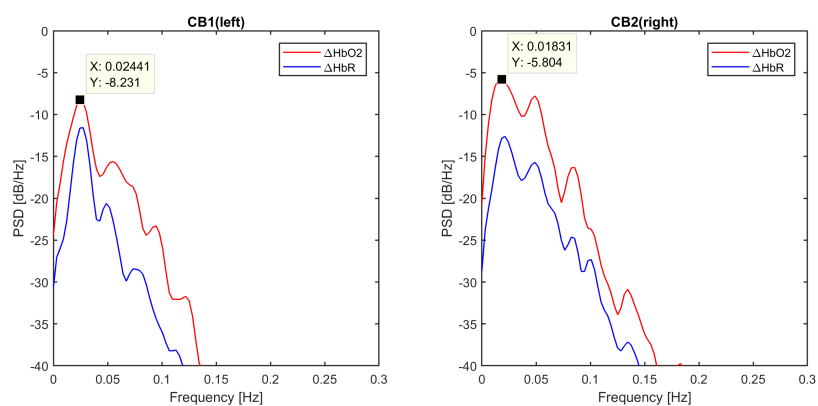

Figure 7. PSD of the left finger tapping signal from S3

\section{DISCUSSION}

fNIRS proved to be a good alternative for its high sampling frequency up to $50 \mathrm{~Hz}$, considering that fMRI allows only to obtain bona-fide 3D images approximately every 4 seconds. On the other side, for fNIRS, the better temporal resolution is obtained at the price of lower spatial resolution compared to fMRI. Anyhow, the purpose of that study was to evaluate whether fNIRS signal is reliable enough to properly assess cerebellar processes. Initially, it is essential to ensure the measurement of meaningful biological signals truly related to cerebellar activity. Afterwards, given the lack of standardization even for the use of fNIRS on the brain [12], many issues remain to be solved, starting from the acquisition part to subsequent signal processing methods needed. From this study, we obtained marked asymmetries in activation among subjects with different levels of lateralization. While the strong ipsilateral activation with the dominant hand movement is in line with previous fMRI studies, the variability of the contralateral behavior between-subjects, also observed in this fMRI study [13], calls for further investigation. The stimulus-induced peaks latencies are higher than the ones reported in literature for the brain. It is sure that the onset time for a hemodynamic response varies across individuals and across brain regions, depending also on type of tasks and protocol design, and participants' age [7], but it will be worth investigating further this aspect. Our hypothesis is that, due to the dense cerebellar vascularization, the BOLD effect might have a different manifestation than in the brain: the measured signal could be more related to a recovery process after a neural activation, rather than to the activation itself. This could also explain the lack of asymmetry in some of the responses (see S1 Fig. 5), as the blood supply for the cerebellum is not as localized as for the brain [14]. On the practical side, no predefined caps with default cerebellar locations are currently available on the market, thus we had to design our own cerebellar cap, to fine-tune the optodes placement and optimize the holders. Moreover, the experimental protocol must be carefully defined: the stimulation frequency does not have to fall within the range of the physiological oscillations that will be removed by means of filtering $(>0.1 \mathrm{~Hz})$, and the minimum time to elicit a hemodynamic response $(>5 \mathrm{~s})$ must be respected with an inter-stimulus-interval sufficient for recovery.

\section{CONCLUSION}

fNIRS was assessed for its capability to measure cerebellar hemodynamics during a finger tapping task. This technique revealed marked differences among subjects with different levels of lateralization. As such, it appears as a promising tool to explore the cerebellum hemodynamics at a finer timescale than by fMRI. Future extended experiments are planned to study motor and cognitive functions through multimodal integration with EEG recordings in multiple settings. This work paves the ground towards the implementation and validation of a new diagnostic tool which is crucial for the interpretation of cerebellar activation patterns and the correlations of lesions and symptoms, especially for particularities in neurology.

\section{REFERENCES}

[1] G.M. Shepherd, "Cerebellum," in The Synaptic Organization of the Brain, 5th ed. Oxford university press, 2004, ch. 7, p. 271.

[2] G.P. Argyropoulos, et al., "The cerebellar cognitive affective/Schmahmann syndrome: a task force paper," The Cerebellum, vol. 19, pp. 102-125, Feb 2020.

[3] X. Guell, and J. Schmahmann, "Cerebellar functional anatomy: a didactic summary based on human fMRI evidence," The Cerebellum, vol. 19, pp. 1-5, Feb 2020.

[4] L.M. Andersen, K. Jerbi, and S.S. Dalal, "Can EEG and MEG detect signals from the human cerebellum?," NeuroImage, vol. 215, article 116817, Jul 2020

[5] P. Pinti, et al., "The present and future use of functional near-infrared spectroscopy (fNIRS) for cognitive neuroscience," Ann. NY Acad. Sci., vol. 1464. no. 1, pp. 5-29, Mar 2020.

[6] G. Rocco, et al., "Exploring the cerebellum with functional nearinfrared imaging: a preliminary study," in I. S. Biomed. Imaging, Apr 2021

[7] K. Tai, and T. Chau, "Single-trial classification of NIRS signals during emotional induction tasks: towards a corporeal machine interface," $J$. Neuroeng. Rehabil., vol. 6, article 39, Nov 2009.

[8] R. C. Oldfield, "The assessment and analysis of handedness: the Edinburgh inventory," Neuropsychologia, vol. 9, no.1, pp. 97-113, Mar 1971.

[9] N.P. Todd, S. Govender, and J.G. Colebatch, "The human electrocerebellogram (ECeG) recorded non-invasively using scalp electrodes," Neurosci. Lett., vol. 682, pp. 124-131, Aug 2018.

[10] R. Oostenveld, et al., "FieldTrip: open source software for advanced analysis of MEG, EEG, and invasive electrophysiological data," Comput. Intel. Neuro., vol. 2011, article 156869, 2011.

[11] D.T. Delpy, and M. Cope, "Quantification in tissue near-infrared spectroscopy," Philos. Trans. R. Soc. B. Biol. Sci., vol. 352, pp. 649659, Jun 1997.

[12] P. Pinti, et al., "Current status and issues regarding pre-processing of fNIRS neuroimaging data: an investigation of diverse signal filtering methods within a general linear model framework," Front. Hum. Neurosci., vol. 12, no. 505, Jan 2019.

[13] L. Jäncke, et al., "The effect of finger-movement speed of the dominant and the subdominant hand on cerebellar activation: a functional magnetic resonance imaging study," Neuroimage, vol. 9, no. 5, pp.497507, May 1999.

[14] A. L. Rhoton, "The Cerebellar Arteries," Neurosurgery, vol. 47, no. suppl. 3, pp. S29-S68, Sep 2000. 